\title{
Generalized topologies with associating function and logical applications
}

\author{
TOMAsz WiTcZAK
}

\begin{abstract}
The whole universe of a generalized topological space may not be open. Hence, some points may be beyond any open set. In this paper we assume that such points are associated with certain open neighbourhoods by means of a special function $\mathcal{F}$. We study various properties of the structures obtained in this way. We introduce the notions of $\mathcal{F}$-interior and $\mathcal{F}$-closure and we discuss issues of convergence in this new setting. It is possible to treat our spaces as a semantical framework for modal logic.
\end{abstract}

\section{Introduction}

In some sense, the whole idea of generalized topological spaces is not new. In fact, it traces back to the beginning of twentieth century, when Moore [15] introduced families of subsets (later known as Moore families) which were closed under intersections and contained the whole universe. McKinsey and Tarski [21] used so-called closure operator to describe very natural properties of logical consequence. There is a strict correspondence between such operators and Moore families. Moreover, one can discuss operators and families which are dual to the ones in question. These dual notions can be considered as weak forms of topological interior and open set. If we say "weak", then it means two things. First, such family does not need to be closed under finite intersections (but still has to be closed under arbitrary unions). Second, the whole universe may not be open.

Received October 4, 2019.

2010 Mathematics Subject Classification. Primary 54A05, 54A20; Secondary 03B45.

Key words and phrases. Generalized topology, modal logic, convergence spaces, nets and sequences.

https://doi.org/10.12697/ACUTM.2020.24.17 
This concept of generalization came back from time to time ${ }^{1}$. For example, in 1960s several authors (N. Levine among them) contributed to the theory of $\alpha$-, semi-, pre-, b- and $\beta$-open sets (see [17]). Each of these families (when considered in the context of ordinary topological spaces) behaves like a generalized topology. In 1980s Masshour et al. [14] introduced supratopological spaces which seem to be identical with strong generalized topological spaces, defined few years later by Császár in [6] and [5].

Probably it was Császár, who has started very systematic investigation of generalized spaces (at least under this name). Many authors followed this line of research. As a result, nowadays we have generalized analogues of almost all basic topological notions: separation axioms (see [7], [9] and [19]), filters (see [16]), convergence (see [2], [3] and [18]) or topological groups (see [11]). Moreover, these spaces are applied in other branches of science (like formal concept analysis and data clustering), where they are known as extensional abstractions (see [20]) or knowledge spaces (see [10]). In addition, a group of Polish authors used the fact that two definitions of nowhere density $^{2}$, which are equivalent in (ordinary) topological spaces, are not equivalent in the generalized framework. This distinction was helpful for them in their research about Banach games and Baire spaces (see [13]).

Recently, we have prepared generalized topological semantics for certain weak modal and subintuitionistic logics (see [22]). One thing should be noted to avoid confusion. Tarski used generalized notion (of closure) to speak about consequence. It means that if we have a set $\mathfrak{F}$ of formulas then $\varphi \in C l(\mathfrak{F})$ if and only if there is a finite subset $\left\{\gamma_{1}, \ldots, \gamma_{n}\right\} \subseteq \mathfrak{F}$ such that $\left\{\gamma_{1}, \ldots \gamma_{n}\right\}=\varphi$ (where $=$ means semantic consequence but we can also use the syntactic consequence symbol $\vdash$ ). On the other hand, he used standard topological interior in the context of modal logic. But in this case he referred not to the "structure of deduction", but to the valuation of $\square \varphi$. Namely, he assumed that if topological space is a model for modal logic, then $V(\square \varphi)=\operatorname{Int}(V(\varphi))$. And this is what interests us primarily: to replace this kind of interior by the generalized one.

While working on this topic, we have developed some purely topological tools which seemed to be interesting and quite natural but beyond the primary scope of our logical research. Hence, they are investigated in the present paper. We assume that each point $w$ which is beyond the maximal open set $\bigcup \mu$ can be associated with certain (possibly non-empty) family $\mathcal{F}_{w}$

\footnotetext{
${ }^{1}$ It should be noted that there are various ways of understanding the notion of generalization. For example, Grothendieck topologies have almost nothing to do with our considerations. Also we do not deal with Császár syntopogenous spaces or Száz's relator spaces, at least not directly or openly. So-called minimal structures and (generalized) weak structures are closer to our intuition but more vague.

${ }^{2}$ The first one assumes that $\operatorname{Int}(C l(A))=\emptyset$. The second one says that $A$ is nowhere dense if and only if for any $G \in \mu$ there is $H \in \mu$ such that $H \subseteq G$ and $A \cap H=\emptyset$.
} 
of open neighbourhoods. This approach allows us to speak about new types of convergence and "openess". In conclusion, we state that each GT $\mathcal{F}$-space (in which $\mathcal{F}_{w} \neq \emptyset$ for any $w \in W$ ) corresponds to some generalized topology in which $W$ is open.

The reader should remember that this particular paper contains auxilliary results and does not deal directly with logic (except Section 3 which rather has a survey character).

\section{General overview of GTF $\mathcal{F}$-spaces}

2.1. Basic notions. First of all, we repeat the very definition of generalized topological space (see [5] and [6]).

Definition 2.1. Assume that $W$ is a non-empty set (universe) and $\mu \subseteq$ $P(W)$. We say that $\mu$ is a generalized topology on $W$ if $\emptyset \in \mu$ and $\mu$ is closed under arbitrary unions, i.e., if $J \neq \emptyset$ and for each $i \in J, X_{i} \in \mu$, then $\bigcup_{i \in J} X_{i} \in \mu$.

In such a case we say that $\langle W, \mu\rangle$ is a generalized topological space. The elements of $\mu$ are named $\mu$-open sets (or just open sets, if there is no risk of confusion) and for any $A \subseteq W$, we define $\operatorname{Int}(A)$ as the union of all open sets contained in $A$.

Sometimes we shall say that all points from $W \backslash \bigcup \mu$ are orphaned. As for the notion of closed set, we say that the set $A \subseteq W$ is closed if its complement is open. We define $C l(A)$ (closure of $A$ ) as the smallest closed set containing $A$. It is easy to show that $C l(A)=W \backslash \operatorname{Int}(W \backslash A)$ (see [8]). Also $w \in C l(A)$ if and only if for any $G \in \mu$ such that $w \in G, G \cap A \neq \emptyset$.

Below we have three examples of infinite $\mathbf{G T}$-spaces (taken from [19], [2]).

(1) $W$ is arbitrary, $\emptyset \neq X \subseteq W, \mu=\{A \subseteq W ; A \subseteq W \backslash X\}$.

(2) $W=\mathbb{R}, \mu=\{\emptyset\} \cup\{A \subseteq \mathbb{R} ; A \backslash\{w\} \subseteq A$ for certain $w \in \mathbb{R}\}$.

(3) $|W|>\omega_{0}, v \notin W, W^{*}=W \cup\{v\}, \mu=\{\emptyset,\{v\} \cup\{W \backslash A\} ; A \subseteq$ $\left.W,|A| \leq \omega_{0}\right\}$, our space is $\left\langle W^{*}, \mu\right\rangle$.

The second thing to do is to establish our new structure, equipped with an additional function which connects orphaned points with open neighbourhoods.

Definition 2.2. We define GTF $\mathcal{F}$-structure as a triple $M_{\mu}=\langle W, \mu, \mathcal{F}\rangle$ such that $\mu$ is a generalized topology on $W$ and $\mathcal{F}$ is a function from $W$ into $P(P(\bigcup \mu))$ such that:

- if $w \in \bigcup \mu$, then $\left[X \in \mathcal{F}_{w} \Leftrightarrow X \in \mu\right.$ and $\left.w \in X\right]\left[\mathcal{F}_{w}\right.$ is a shortcut for $\mathcal{F}(w)]$;

- if $w \in W \backslash \bigcup \mu$, then $\left[X \in \mathcal{F}_{w} \Rightarrow X \in \mu\right]$.

One can say that $\mathcal{F}$ is an arbitrary extension (on the whole $W$ ) of the map that associates with any $w \in \bigcup \mu$ the principal filter generated by $w$. 
The next definition is just a useful shortcut.

Definition 2.3. Assume that $\langle W, \mu, \mathcal{F}\rangle$ is a $\mathbf{G T F} \mathcal{F}$-structure and $A \in \mu$. Then we introduce the following notation: $A^{-1}=\left\{z \in W ; A \in \mathcal{F}_{z}\right\}$.

Below we shall discuss a simple example of $\mathbf{G T} \mathcal{F}$-structure. Its basic form is based strictly on Example 3.1 from [2].

Example 2.4. Consider $\langle W, \mu, \mathcal{F}\rangle$, where:

$W=\mathbb{Z}, \mu=\{\emptyset,\{1\},\{1,3\},\{1,3,5\},\{1,3,5,7\}, \ldots\}, \mathcal{F}_{n}=\emptyset$ for any $n \in$ $2 \mathbb{Z}$. Note that if $n$ is odd, then (by means of general definition) $\mathcal{F}_{n}$ is just a collection of its open neighbourhoods.

Of course, this is a $\mathbf{G T} \mathcal{F}$-structure, but undoubtedly it is a rather degenerated case. However, we may replace $\mathcal{F}$ by:

(1) $\mathcal{F}^{\prime}$. Consider $\gamma: 2 \mathbb{Z} \rightarrow 2 \mathbb{Z}+1$, where $\gamma(x)=\max \{m ; m \in 2 \mathbb{Z}+1, m<$ $x\}$. Assume that:

- if $n \in 2 \mathbb{Z}+1$, then $G \in \mathcal{F}_{m}^{\prime} \Leftrightarrow G \in \mu$ and $m \in G$;

- if $n \in 2 \mathbb{Z}$, then $G \in \mathcal{F}_{n}^{\prime} \Leftrightarrow G \in \mathcal{F}_{\gamma(n)}^{\prime}$.

For example, $\mathcal{F}_{8}^{\prime}=\mathcal{F}_{\gamma(8)}^{\prime}=\mathcal{F}_{7}^{\prime}=\{\{1,3,5,7\},\{1,3,5,7,9\},\{1,3,5$, $7,9,11\}, \ldots\}$

(2) $\mathcal{F}^{\prime \prime}$. It is just like $\mathcal{F}^{\prime}$ but instead of $\gamma$ we use $\delta(x)=\min \{m ; m \in$ $2 \mathbb{Z}+1, m>x\}$. Then $\mathcal{F}_{8}^{\prime \prime}=\{\{1,3,5,7,9\},\{1,3,5,7,9,11\}, \ldots\}$.

(3) $\mathcal{F}^{\prime \prime \prime}$. We use $\gamma$ again and if $n \in 2 \mathbb{Z}$, then we define

$$
G \in \mathcal{F}_{n}^{\prime \prime \prime} \Leftrightarrow G \in \mu, G \neq \emptyset \text { and } G \notin \mathcal{F}_{\gamma(n)+2}^{\prime \prime \prime} .
$$

Now $\mathcal{F}_{8}^{\prime \prime \prime}=\{\{1\},\{1,3\},\{1,3,5\},\{1,3,5,7\}\}$.

$\mathcal{F}$ can be very arbitrary but the most interesting cases are those with certain regularities. Later (when discussing themes of logic and convergence) we shall come back to this question. It is fruitful to assume that each point from $W \backslash \bigcup \mu$ is associated with a certain point from $\bigcup \mu$ and inherits its neighbourhoods.

2.2. $\mathcal{F}$-interiors and $\mathcal{F}$-open sets. The notions of $\mathcal{F}$-interior and $\mathcal{F}$ closure are based on the intuition arising from the typical understanding of openness and closedness. However, one must note that there will be no full analogy. This situation can be considered both as a limitation and a strength.

Definition 2.5. Let $\langle W, \mu, \mathcal{F}\rangle$ be a $\mathbf{G T} \mathcal{F}$ and $w \in W$. Assume that $A \subseteq W$. We say that $w \in \mathcal{F} \operatorname{Int}(A)$ if there is $G \in \mathcal{F}_{w}$ such that $G \subseteq A$.

The basic properties of $\mathcal{F}$ Int are summarized in the following lemmas (we omit most of the proofs because they are simple).

Lemma 2.6. Assume that $A, B \subseteq W$ and $A \subseteq B$. Then $\mathcal{F}$ Int $(A) \subseteq$ $\mathcal{F} \operatorname{Int}(B)$. 
Lemma 2.7. Assume that $A \subseteq W$. Then $\operatorname{Int}(A) \subseteq \mathcal{F} \operatorname{Int}(A)$. In particular, if $A \in \mu$, then $A \subseteq \mathcal{F} \operatorname{Int}(A)$.

Lemma 2.8. If $A \subseteq W$, then $\mathcal{F} \operatorname{Int}(A) \cap \bigcup \mu=\operatorname{Int}(A)$.

Proof. ( $\subseteq$ ) If $v \in \mathcal{F} \operatorname{Int}(A) \cap \bigcup \mu$, then there is $G \in \mathcal{F}_{v}$ such that $G \subseteq A$. Of course, $v \in G$ (because $v \in \bigcup \mu$ ). Hence, $v \in \operatorname{Int}(A)$.

() If $v \in \operatorname{Int}(A)$, then $v \in \mathcal{F} \operatorname{Int}(A)$ (by means of Lemma 2.7). But if $v \in \operatorname{Int}(A)$, then $v \in \bigcup \mu$. Hence, $v \in \mathcal{F} \operatorname{Int}(A) \cap \bigcup \mu$.

Lemma 2.9. Assume that $A \subseteq \mathcal{F} \operatorname{Int}(A) \subseteq \bigcup \mu$. Then $A \in \mu$.

Lemma 2.10. If $A \subseteq W$, then $\mathcal{F} \operatorname{Int}(\mathcal{F} \operatorname{Int}(A)) \cap \bigcup \mu \subseteq \mathcal{F} \operatorname{Int}(A)$.

Lemma 2.11. Assume that $A \in \mu$. Then $A^{-1} \subseteq \mathcal{F} \operatorname{Int}(A)$.

Lemma 2.12. $\mathcal{F} \operatorname{Int}(W)=W \Leftrightarrow$ for any $w \in W, \mathcal{F}_{w} \neq \emptyset$.

Proof. $(\Rightarrow)$ Assume that there is $v \in W$ such that $F_{v}=\emptyset$. Then $v \notin$ $\mathcal{F} \operatorname{Int}(W)$.

$(\Leftarrow)$ Assume that $v \notin \mathcal{F} \operatorname{Int}(W)$. This means that for any $G \in \mathcal{F}_{v}, G \nsubseteq W$. Clearly, this is possible only if there are no any sets in $\mathcal{F}_{v}$.

Lemma 2.13. $\mathcal{F}$ Int $(\emptyset)=\emptyset \Leftrightarrow$ for any $w \in W, \emptyset \notin \mathcal{F}_{w}$.

Proof. The proof is similar to the former one.

Lemma 2.14. Assume that for certain $X \subseteq W$ and for any $A \in \mu$ : if $A \neq \emptyset$, then $A \nsubseteq X$. Then $\mathcal{F} \operatorname{Int}(X)=\emptyset$ or $\mathcal{F} \operatorname{Int}(X) \subseteq Z=\{z \in W ; \emptyset \in$ $\left.\mathcal{F}_{z}\right\}$.

Note that if there is at least one $X \subseteq W$ such that $\mathcal{F} \operatorname{Int}(X)=\emptyset$, then $Z$ (defined as above) must be empty.

As for the Definition 2.5, it is modeled after the standard definition of interior in (generalized or not) topological spaces. Note, however, that in general we cannot say that $\mathcal{F} \operatorname{Int}(A) \subseteq A$. To see this $^{3}$, it is sufficient to consider any $A \in \mu$ and $w \in W \backslash \bigcup \mu$ such that $A \in \mathcal{F}_{w}$. Clearly, $w \in$ $\mathcal{F} \operatorname{Int}(A)$ but $w \notin A$. What is less surprising is that sometimes $A \nsubseteq \mathcal{F} \operatorname{Int}(A)$. Note that in such situation $A \backslash \mathcal{F} \operatorname{Int}(A) \subseteq A \cap(W \backslash \bigcup \mu)$.

For the reasons above, it is sensible to consider at least three concepts related to the notion of openness.

Definition 2.15. Let $\langle W, \mu\rangle$ be a $\mathbf{G T F}$ and $A \subseteq W$. We say that $A$ is:

- $\mathcal{F}$-open $\left(\mathcal{F}_{\text {o. }}\right)$ if $A=\mathcal{F} \operatorname{Int}(A)$;

- $\mathrm{d} \mathcal{F}$-open $\left(\mathrm{d} \mathcal{F}_{\mathrm{o}}\right.$. $)$ if $\mathcal{F} \operatorname{Int}(A) \subseteq A$;

- $\mathrm{u} \mathcal{F}$-open $(\mathrm{u} \mathcal{F}$ o. $)$ if $A \subseteq \mathcal{F} \operatorname{Int}(A)$.

\footnotetext{
form.

${ }^{3}$ Sometimes our examples and counter-examples will be presented only in a sketchy
} 
The next two lemmas give us certain information about arbitrary unions and intersections of $\mathcal{F}$-interiors.

Lemma 2.16. Suppose that $J \neq \emptyset$ and $\left\{X_{i}\right\}_{i \in J}$ is a family of subsets of $W$. Then $\bigcup_{i \in J} \mathcal{F} \operatorname{Int}\left(X_{i}\right) \subseteq \mathcal{F} \operatorname{Int}\left(\bigcup_{i \in J} X_{i}\right)$. If each $X_{i}$ is $u \mathcal{F}$ o., then $\bigcup_{i \in J} X_{i} \subseteq \mathcal{F} \operatorname{Int}\left(\bigcup_{i \in J} X_{i}\right)$.

Proof. Let $v \in \bigcup_{i \in J} \mathcal{F} \operatorname{Int}\left(X_{i}\right)$. Hence, there is $k \in J$ such that $v \in$ $\mathcal{F} \operatorname{Int}\left(X_{k}\right)$. Then there is $G \in \mathcal{F}_{v}$ such that $G \subseteq X_{k}$. But then $G \subseteq X_{k} \subseteq$ $\bigcup_{i \in J} X_{i}$. Therefore, we can say that $v \in \mathcal{F} \operatorname{Int}\left(\bigcup_{i \in J} X_{i}\right)$.

Note that we can easily imagine the following situation: there is $v \in W$ such that for any $G \in \mathcal{F}_{v}$ and for each $i \in J, G \nsubseteq \mathcal{F} \operatorname{Int}\left(X_{i}\right)$ but at the same time there is $H \in \mathcal{F}_{v}$ such that $H \subseteq \bigcup_{i \in J} \mathcal{F} \operatorname{Int}\left(X_{i}\right)$. Hence, $v \in$ $\mathcal{F} \operatorname{Int}\left(\bigcup_{i \in J} X_{i}\right)$ but $v \notin \bigcup_{i \in J} \mathcal{F} \operatorname{Int}\left(X_{i}\right)$. For example, consider $W=\mathbb{R}^{2}$ with standard (hence, in particular, generalized) topology $\mu$ limited only to the ball $K[(0,0), r], r>0$ (i.e., $K=\bigcup \mu$ ). Then we may take any $v$ outside $K$, two arbitrary sets $X_{1}, X_{2}$ (both contained in $K$ ) and any set $B$ contained in $X_{1} \cup X_{2}$ but not in $X_{1}$ nor in $X_{2}$. Now it is enough to assume that $B \in \mathcal{F}_{v}$ and for any set $C \in \mu$ : if $C \subseteq X_{1}$ or $C \subseteq X_{2}$, then $C \notin \mathcal{F}_{v}$.

Lemma 2.17. Suppose that $J \neq \emptyset$ and $\left\{X_{i}\right\}_{i \in J}$ is a family of subsets of $W_{\mu}$. Then $\mathcal{F} \operatorname{Int}\left(\bigcap_{i \in J} X_{i}\right) \subseteq \bigcap_{i \in J} \mathcal{F} \operatorname{Int}\left(X_{i}\right)$. If each $X_{i}$ is $d \mathcal{F}$ o., then $\mathcal{F} \operatorname{Int}\left(\bigcap_{i \in J} X_{i}\right) \subseteq \bigcap_{i \in J} X_{i}$.

Proof. The proof is similar to the former one. Also we can find a counterexample for the opposite inclusion.

As we said, $\mathcal{F}$-open sets do not have all the properties of open sets, even in Császár's sense. Nonetheless, the following theorem can be considered as useful. In fact, it will be useful in our further investigations.

Theorem 2.18. Let $\langle W, \mu, \mathcal{F}\rangle$ be a $\mathbf{G T} \mathcal{F}$-structure and $w \in W$. Then $\mathcal{F}_{w} \neq \emptyset$ if and only if there is $\mathcal{F}_{o}$. set $G \subseteq W$ such that $w \in G$.

Proof. Necessity. Since $\mathcal{F}_{w} \neq \emptyset$, there is at least one $A \in \mathcal{F}_{w}$. Of course, $w \in \mathcal{F} \operatorname{Int}(A)$. If $A=\mathcal{F} \operatorname{Int}(A)$, then we can finish our proof. If not, then it means that $\mathcal{F} \operatorname{Int}(A) \nsubseteq A$. Let us define $G$ as $A \cup \mathcal{F} \operatorname{Int}(A)$. We show that $G$ is open, i.e., that $\mathcal{F} \operatorname{Int}(G)=G$.

$(\subseteq)$ Let $v \in \mathcal{F} \operatorname{Int}(G)$. Hence $v \in \mathcal{F} \operatorname{Int}(A \cup \mathcal{F} \operatorname{Int}(A))$. Now there is $U \in \mathcal{F}_{v}$ such that $U \subseteq A \cup \mathcal{F} \operatorname{Int}(A)$. In fact, it means that $U \subseteq A$ (because $U \subseteq \bigcup \mu$ and $\mathcal{F} \operatorname{Int}(A) \cap \bigcup \mu=\operatorname{Int}(A)=A)$. Thus $v \in \mathcal{F} \operatorname{Int}(A)$. From this we infer that $v \in G$.

$(\supseteq)$ Let $v \in G$. Hence $v \in A$ or $v \in \mathcal{F} \operatorname{Int}(A)$. If $v \in A$, then $A \in \mathcal{F}_{v}$ (because $A \in \mu$ ). Therefore $v \in \mathcal{F} \operatorname{Int}(G)$. If $v \in \mathcal{F} \operatorname{Int}(A)$, then there is $U \in \mathcal{F}_{v}$ such that $U \subseteq A \subseteq G$. Thus $v \in \mathcal{F} \operatorname{Int}(G)$. 
Sufficiency. Suppose that $G \subseteq W$ is $\mathcal{F}_{o} ., w \in G$ and $\mathcal{F}_{w}=\emptyset$. Of course $\mathcal{F} \operatorname{Int}(G)=G$, so $w \in \mathcal{F} \operatorname{Int}(G)$. Hence there is $H \in \mathcal{F}_{w}$ such that $H \subseteq G$, a contradiction.

Note that in the case of $\Leftarrow$-direction it is enough to assume that $G$ is $u \mathcal{F}_{\text {o. }}$

2.3. $\mathcal{F}$-closures and $\mathcal{F}$-closed sets. Any sensible definition of "openess" should be dual to certain understanding of "closedness". We propose the following notion, based on the very well known property of closed (and generalized closed) sets.

Definition 2.19. Let $\langle W, \mu, \mathcal{F}\rangle$ be a $\mathbf{G T} \mathcal{F}$ and $w \in W$. Assume that $A \subseteq W$. We say that $w \in \mathcal{F} C l(A)$ if for any $G \in \mathcal{F}_{w}, G \cap A \neq \emptyset$.

Now we can define $\mathcal{F}$-closed sets.

Definition 2.20. Let $\langle W, \mu, \mathcal{F}\rangle$ be a $\mathbf{G T} \mathcal{F}$ and $A \subseteq W$. We say that $A$ is:

- $\mathcal{F}$-closed $(\mathcal{F}$ c. $)$ if $\mathcal{F} C l(A)=A$;

- $\mathrm{d} \mathcal{F}$-closed $(\mathrm{d} \mathcal{F}$ c. $)$ if $\mathcal{F} C l(A) \subseteq A$;

- $\mathrm{u} \mathcal{F}$-closed (uF $\mathcal{F}_{\mathrm{c}}$ ) if $A \subseteq \mathcal{F} C l(A)$.

This definition makes sense because it gives us expected dualism.

Theorem 2.21. Let $\langle W, \mu, \mathcal{F}\rangle$ be a $\mathbf{G T} \mathcal{F}$. Assume that $A \subseteq W$ is $\mathcal{F}$ open. Then the set $-A$ is $\mathcal{F}$-closed.

Proof. We know that $\mathcal{F} \operatorname{Int}(A)=\left\{z \in W\right.$; there is $G \in \mathcal{F}_{z}$ such that $G \subseteq$ $A\}=A$. Let us consider $-A=\left\{z \in W\right.$; for each $\left.G \in \mathcal{F}_{z}, G \nsubseteq A A\right\}$. We shall show that $\mathcal{F} C l(-A)=-A$.

$(\subseteq)$ Assume that $w \in \mathcal{F} C l(-A)$. Hence, for any $G \in \mathcal{F}_{w}, G \cap-A \neq \emptyset$. Now $G \nsubseteq A$ and for this reason $w \in-A$.

() Suppose that $w \in-A$ and assume that there is $H \in \mathcal{F}_{w}$ such that $H \cap-A=\emptyset$. It means that $H \subseteq A$. But then $w \in \mathcal{F} \operatorname{Int}(A)=A$ which gives us a plain contradiction.

As in the case of interiors, properties of $\mathcal{F} C l$ are rather weak. For example, we may ask if $A \subseteq \mathcal{F} C l(A)$. The answer is (in general) negative. We may easily imagine the following situation: $A \subseteq W, A \cap(W \backslash \bigcup \mu) \neq \emptyset$, $w \in A \cap(W \backslash \bigcup \mu)$ and there is $G \in \mathcal{F}_{w}$ such that $G \cap A=\emptyset$. On the other hand, it is also possible that $\mathcal{F} C l(A) \nsubseteq \subseteq A$.

We have the following lemmas (with respect to an arbitrary $\langle W, \mu, \mathcal{F}\rangle$ ).

Lemma 2.22. $\mathcal{F} C l(\emptyset)=\emptyset \Leftrightarrow$ for any $z \in W, \mathcal{F}_{z} \neq \emptyset$.

Lemma 2.23. $\mathcal{F} C l(W)=W \Leftrightarrow$ for any $z \in W, \emptyset \notin \mathcal{F}_{z}$.

Clearly, these statements are analogous to the corresponding ones for $\mathcal{F}$ interiors. The same can be said about the following one. 
Lemma 2.24. Suppose that $J \neq \emptyset$ and $\left\{X_{i}\right\}_{i \in J}$ is a family of subsets of $W$. Then $\bigcup_{i \in J} \mathcal{F} C l\left(X_{i}\right) \subseteq \mathcal{F} C l\left(\bigcup_{i \in J} X_{i}\right)$. If each $X_{i}$ is $u \mathcal{F} c$., then $\bigcup_{i \in J} X_{i} \subseteq \mathcal{F} C l\left(\bigcup_{i \in J} X_{i}\right)$.

Proof. Let $v \in \bigcup_{i \in J} \mathcal{F C l}(A)$. It means that there is $k \in J$ such that $v \in \mathcal{F} C l\left(X_{k}\right)$. Hence, for any $G \in \mathcal{F}_{v}, G \cap X_{k} \neq \emptyset$. Clearly, $X_{k} \subseteq \bigcup_{i \in J} X_{i}$. Thus $G \cap \bigcup_{i \in J} X_{i} \neq \emptyset$. For this reason, $v \in \mathcal{F} C l\left(\bigcup_{i \in J} X_{i}\right)$.

Similarily to the case of $\mathcal{F}$-interiors, we can find a counter-example for the opposite inclusion. As for the next lemma, it is about intersections.

Lemma 2.25. Suppose that $J \neq \emptyset$ and $\left\{X_{i}\right\}_{i \in J}$ is a family of subsets of $W_{\mu}$. Then $\mathcal{F} C l\left(\bigcap_{i \in J} X_{i}\right) \subseteq \bigcap_{i \in J} \mathcal{F} C l\left(X_{i}\right)$. If each $X_{i}$ is $d \mathcal{F} c$., then $\mathcal{F} C l\left(\bigcap_{i \in J} X_{i}\right) \subseteq \bigcap_{i \in J} X_{i}$.

As earlier, the converse is not true (in general). Finally, we should prove quite simple but important lemma (which will be used later).

Lemma 2.26. Assume that $\langle W, \mu, \mathcal{F}\rangle$ is a $\mathbf{G T}$-frame and $A \subseteq W$. Then $\mathcal{F} C l(A) \subseteq C l(A)$.

Proof. Let us assume that $w \in \mathcal{F} C l(A)$. Hence, for any $G \in \mathcal{F}_{w}, G \cap A \neq$ $\emptyset$. If $w \in \bigcup \mu$, then for each $G \in \mathcal{F}_{w}, G \in \mu$ and $w \in G$. Hence $w \in C l(A)$. If $w \in W \backslash \bigcup \mu$, then for each $G \in \mu, w \notin G$. Thus our conclusion is trivially true.

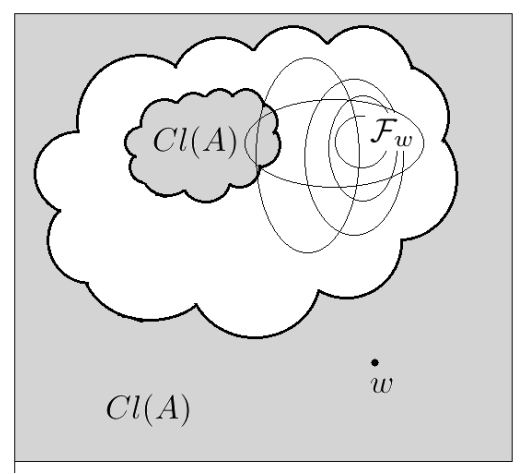

Figure 1. $w \notin \mathcal{F} C l(A)$ but $w \in C l(A)$

The converse is not true, as Figure 1 pictures it. Clearly, $w \in W \backslash \operatorname{Int}(W \backslash$ $A)=C l(A)$ but at the same time there are some sets in $\mathcal{F}_{w}$ which have empty intersection with $A$. 


\section{Some logical applications}

In [22] we have used $\mathbf{G T}$-frames and $\mathbf{G T} \mathcal{F}$-frames as semantical models. The whole idea was based, as usual, on the concept of possible worlds. For example, in case of $\mathbf{G T} \mathcal{F}$-frames forcing of necessity in a given world $w \in W$ was defined as follows:

$w \Vdash_{\mu} \square \varphi \Leftrightarrow$ there is $\mathcal{O}_{w} \in \mathcal{F}_{w}$ such that for each $v \in \mathcal{O}_{w}, v \Vdash \varphi$.

Our GT-models are complete with respect to the logics MT4 and MNT4, the latter refers to the case of strong spaces. This was also proved by Järvinen et al. [12] in terms of interior systems. These authors were motivated by certain reflexions on the approximate reasoning and rough sets. Also, our models turned out to be similar to the (complete) extensional abstractions, investigated by Soldano in [20].

Moreover, we adopted the notion of impossible worlds, i.e., worlds in which "everyting is possible and nothing is necessary" (that is, $\diamond \varphi$ holds for any $\varphi$ and $\square \varphi$ fails for any $\varphi$ ). If we assume that for any $w \in W \backslash \bigcup \mu, \mathcal{F}_{w}=\emptyset$, then these worlds are impossible. Finally, we used strong GT-frames as a sound semantics for certain subintuitionistic logic. While intuitionism does not contain the law of the excluded middle, subintuitionistic logics are even weaker (for example, they may not contain modus ponens rule or a fortiori axiom).

We did not obtain completeness result for $\mathbf{G T} \mathcal{F}$-structures. However, we have investigated similar but more concrete class of GTf -frames. They consist of two subsets: each world from $Y_{1}$ is connected (by means of a special function $\mathbf{f}$ ) with certain world from $\bigcup \mu$ and inherits its family of open neighbourhoods; while each world from $Y_{2}$ has its own family $\mathcal{N}_{w}$ of neighbourhoods. In this environment we defined forcing of two modal operators $\square$ and $\boldsymbol{\square}$. We obtained completeness for two systems based on these modalities.

It is known that certain (ordinary) topological properties have their logical characterizations. Other properties are not definable, at least in a standard propositional modal language. It would be cognitively valuable to obtain analogous characterizations in the generalized framework. Some attempts of this sort have been made in the context of so-called peritopological spaces, investigated by Ahmet and Mehmet [1]. A peritopology on a given set $W$ is a family $(\mathcal{V}(w))_{w \in W}$ where each $\mathcal{V}(w)$ is either a proper filter on $W$ or the unproper filter, e.g. the whole $P(W)$. For example, the classes of $T_{0}, T_{1}$ and $T_{2}$-peritopological spaces ${ }^{4}$ are not modal definable.

\footnotetext{
${ }^{4}$ Of course these spaces are not identical with $\mathbf{G T}$-frames.
} 


\section{Generalized nets and sequences}

In this section we adhere mostly to the notions studied by Baskaran et. al in [2] and [3]. Of course, they are placed in our specific environment. Moreover, we have developed some new definitions and ideas. We have been inspired also by the papers [16] and [18].

The first definition refers to the notion of generalized net.

Definition 4.1. Let $\langle W, \mu, \mathcal{F}\rangle$ be a $\mathbf{G T} \mathcal{F}$ and $(P, \geq)$ be a poset. A generalized net (gnet) in $W$ is a function $f: P \rightarrow W$. The image of $\lambda \in P$ under $f$ is denoted by $f_{\lambda}$ and the whole gnet is denoted as $\left(f_{\lambda}\right)$.

Note that generalized net has pre-ordered and not necessarily directed domain (this was assumed both in [2] and [18]). For this reason we can introduce also two other notions.

Definition 4.2. Let $\langle W, \mu, \mathcal{F}\rangle$ be a $\mathbf{G T} \mathcal{F}$ and $(P, \geq)$ be a poset. We say that a gnet $\left(f_{\lambda}\right), f: P \rightarrow W$, is a net if $P$ is directed, i.e., for any two elements $\lambda_{1}, \lambda_{2} \in P$ there is $\lambda_{3} \in P$ such that $\lambda_{1} \leq \lambda_{3}$ and $\lambda_{2} \leq \lambda_{3}$. If $P=\mathbb{N}$, then we say that $\left(f_{\lambda}\right)$ is a sequence.

Now we go to the convergence, using $\mathcal{F}$ directly.

Definition 4.3. Let $\langle W, \mu, \mathcal{F}\rangle$ be a $\mathbf{G T} \mathcal{F}$ and $\left(f_{\lambda}\right)$ be a gnet in $W$. We say that:

- $\left(f_{\lambda}\right)$ is eventually in $U \subseteq W$ if there is $\lambda_{0} \in P$ such that for any $\lambda \geq \lambda_{0}, f_{\lambda} \in U$

- $\left(f_{\lambda}\right)$ converges to $w \in W$ (i.e. $\left(f_{\lambda}\right) \rightarrow w$ ) if for any $G \in \mathcal{F}_{w}, f_{\lambda}$ is eventually in $G$. In this case we say that $w$ is a limit of $\left(f_{\lambda}\right)$. We say that $\left(f_{\lambda}\right)$ is convergent if there is $v \in W$ such that $\left(f_{\lambda}\right) \rightarrow v$;

- $\left(f_{\lambda}\right)$ is frequently in $U$ if for any $\lambda \in P$ there is $\lambda_{1} \in P$ such that $\lambda_{1} \geq \lambda$, we have $f_{\lambda_{1}} \in U$. We say that $w$ is a limit point of $\left(f_{\lambda}\right)$ if it is frequently in every $G \in \mathcal{F}_{w}$.

Sometimes, when it is useful for clarity, we shall name this kind of convergence as $\mathcal{F}$-convergence or $\rightarrow$-convergence. Contrary to the result for GT (without $\mathcal{F}$ ), in our environment constant gnet may not be convergent.

Lemma 4.4. Let $\langle W, \mu, \mathcal{F}\rangle$ be a $\mathbf{G T} \mathcal{F}$ and $\left(f_{\lambda}\right)=(w)$ be a constant gnet in $W$. Then $(w)$ is convergent if and only if $(w) \rightarrow w$.

Proof. Sufficiency. This is obvious.

Necessity. Assume the contrary, i.e., that there is $v \in W, v \neq w$ such that $(w) \rightarrow v$ but $(w) \nrightarrow w$. Hence, for any $G \in \mathcal{F}_{v}, w \in G$ (note that we speak about constant gnet) but there still is $H \in \mathcal{F}_{w}$ such that $w \notin H$. But if $w$ is in each open neighbourhood of $v$, then $w$ must be in $\bigcup \mu$. Then for any $G \in \mathcal{F}_{w}, w \in G$, hence the existence of $H$ is not possible. 
Theorem 4.5. Let $\langle W, \mu, \mathcal{F}\rangle$ be a $\mathbf{G T} \mathcal{F}$ and $\left(f_{\lambda}\right)=(w)$ be a constant gnet in $W$. Then $\left(f_{\lambda}\right)$ is convergent if and only if $w \in \bigcup \mu$ or $\mathcal{F}_{w}=\emptyset$.

Proof. Sufficiency. Assume that $\left(f_{\lambda}\right)$ is not convergent. In particular (by the preceeding lemma) it means that $(w) \nrightarrow w$. Hence, there is $G \in \mathcal{F}_{w}$ such that $w \notin G$. Now we have two options. If $w \in \bigcup \mu$, then $w \in G$, this is a contradiction. If $\mathcal{F}_{w}=\emptyset$ (which means, in particular, that $w \in W \backslash \bigcup \mu$ ), then $w \notin G \subseteq \bigcup \mu$.

Necessity. Now $(w)$ is convergent. In particular, it means that $(w) \rightarrow w$. Suppose that $w \notin \bigcup \mu$ and $\mathcal{F}_{w} \neq \emptyset$. But then for any $G \in \mathcal{F}_{w}, w \notin G$. Hence $\left(f_{\lambda}\right)=(w)$ is not eventually in $G$. Contradiction with convergence.

The next question about constant gnets is: is the limit of a convergent constant gnet unique? Let us introduce a certain subclass of our structures.

Definition 4.6. We say that a $\mathbf{G T} \mathcal{F}$-structure $\langle W, \mu, \mathcal{F}\rangle$ is $\mathcal{F} T_{1}$ if for any $w \neq v$ there are $G \in \mathcal{F}_{w}$ such that $v \notin G$ and $H \in \mathcal{F}_{v}$ such that $w \notin H$.

Theorem 4.7. Let $\langle W, \mu, \mathcal{F}\rangle$ be a $\mathbf{G T} \mathcal{F}$-structure. Then the limit of every constant and convergent gnet is unique if and only if $\langle W, \mu, \mathcal{F}\rangle$ is $\mathcal{F} T_{1}$.

Proof. Necessity. Assume that $\left(f_{\lambda}\right)$ has a unique limit $w$. Hence, for any $v \neq w, f_{\lambda}=(w) \nrightarrow v$. Thus there is $H \in \mathcal{F}_{v}$ such that $w \notin H$.

But maybe for any $G \in \mathcal{F}_{w}, v \in G$ ? This would mean that $(v) \rightarrow w$ (by the very definition of convergence). However, $(v) \rightarrow v$ and the limits are unique, so $v=w$, a contradiction.

Sufficiency. Suppose that our space is $\mathcal{F} T_{1}$. Let $w \neq v$, and $(w)$ be a convergent gnet. Then $(w) \rightarrow w$. Assume that at the same time $(w) \rightarrow v$. It means that for any $G \in \mathcal{F}_{v}, w \in G$. But this is a contradiction.

The following theorem is nearly compatible with $(\Rightarrow)$ part of Theorem 13 in [2]. However, we must assume that our gnet $(w)$ is convergent.

Theorem 4.8. Let $\langle W, \mu, \mathcal{F}\rangle$ be a $\mathbf{G T} \mathcal{F}$. Assume that $w, v \in W, w \neq v$, $(w)$ is a convergent, constant gnet and $f_{\lambda}$ may be an arbitrary gnet (in $W$ ). Then

$\left[\left(f_{\lambda}\right) \rightarrow w \Rightarrow\left(f_{\lambda}\right) \rightarrow v\right] \Rightarrow\left[w \in \bigcap \mathcal{F}_{v}\right]$.

Proof. Suppose that whenever $\left(f_{\lambda}\right) \rightarrow w$, also $\left(f_{\lambda}\right) \rightarrow v$. Let us consider the constant gnet $w, w, w, \ldots$ It converges to $w$ but also to $v$. Hence, $w$ is eventually in every $G \in \mathcal{F}_{v}$. This means that $w \in \cap \mathcal{F}_{v}$.

In the next conclusion we do not need to assume that $(w)$ is convergent. This proposition is just like $(\Leftarrow)$ from the aforementioned theorem.

Theorem 4.9. Let $\langle W, \mu, \mathcal{F}\rangle$ be a $\mathbf{G T} \mathcal{F}$. Assume that $w, v \in W, w \neq v$ and $\left(f_{\lambda}\right)$ is an arbitrary gnet in $W$. Then

$\left[w \in \bigcap \mathcal{F}_{v}\right] \Rightarrow\left[\left(f_{\lambda}\right) \rightarrow w \Rightarrow\left(f_{\lambda}\right) \rightarrow v\right]$. 
Proof. Suppose that $w \in \bigcap \mathcal{F}_{v}$. It allows us to say that any $H \in \mathcal{F}_{v}$ is also in $\mathcal{F}_{w}$. Hence $\mathcal{F}_{v} \subseteq \mathcal{F}_{w}$. Now assume that $\left(f_{\lambda}\right) \rightarrow w$. Thus $\left(f_{\lambda}\right)$ is eventually in every $G \in \mathcal{F}_{w}$. In particular, it is in every $G \in \mathcal{F}_{v}$. Clearly, this means that $\left(f_{\lambda}\right) \rightarrow v$.

The next lemma is an interesting and useful observation.

Lemma 4.10. Let $\langle W, \mu, \mathcal{F}\rangle$ be a $\mathbf{G T} \mathcal{F}$ and $\left(f_{\lambda}\right)$ be a gnet. If $\left(f_{\lambda}\right) \rightarrow$ $w \in W$, then $\emptyset \notin \mathcal{F}_{w}$.

Proof. Assume that $\emptyset \in \mathcal{F}_{w}$. By convergence, we know that for any $G \in$ $\mathcal{F}_{w}$, so also for $\emptyset$, there is $\lambda_{0} \in P$ such that for each $\lambda \geq \lambda_{0}, f_{\lambda} \in \emptyset$. This is impossible.

The last lemma in this section is a modification of Theorem 2.6 in [2].

Lemma 4.11. Let $\langle W, \mu, \mathcal{F}\rangle$ be $a \mathbf{G T F}$ and $f: P \rightarrow W$ be a gnet in $W$. Assume that $m$ is a maximal element of $P$ and $f_{m} \in \bigcup \mu$ or $\mathcal{F}_{f_{m}}=\emptyset$. Then $\left(f_{\lambda}\right) \rightarrow f_{m}$.

Proof. If $f_{m} \in \bigcup \mu$, then let us consider an arbitrary $G \in \mathcal{F}_{f_{m}}$. Of course, $f_{m} \in G$ and $f_{\lambda} \in G$ for each $\lambda \geq m$. The reason is that $\lambda \geq m$ implies $\lambda=m$. We conclude that $f_{\lambda} \rightarrow f_{m}$.

If $\mathcal{F}_{f_{m}}$ is empty, then our result is trivial.

\section{A higher level of convergence}

We have already proved that each point of $W$ is contained in certain $\mathcal{F}$ open neighbourhood (if $\mathcal{F}_{w} \neq \emptyset$ ). This observation leads us to the second understanding of convergence.

Definition 5.1. Let $\langle W, \mu, \mathcal{F}\rangle$ be a $\mathbf{G T} \mathcal{F}$-structure. Assume that $w \in$ $W$. We define $\mathcal{E}_{w}$ as the set of all $\mathcal{F}$-open sets to which $w$ belongs.

As we know from Theorem $2.18, \mathcal{E}_{w}=\emptyset$ if and only if $\mathcal{F}_{w}=\emptyset$. Let us go back to the $\mathbf{G T} \mathcal{F}$-structure from Example 2.4.

Example 5.2. Recall that we are working with $\langle\mathbb{Z}, \mu, \mathcal{F}\rangle$, where $\mu=$ $\{\emptyset,\{1\},\{1,3\},\{1,3,5\},\{1,3,5,7\}, \ldots\}$. Let $m$ be an odd integer. Consider an arbitrary $G \in \mathcal{F}_{m}$. We assumed that for any $n \in 2 \mathbb{Z}, \mathcal{F}_{n}=\emptyset$. For this reason, $\mathcal{F} \operatorname{Int}(G) \subseteq G$. By means of Lemma 2.7 and the fact that $G \in \mu, G$ is $\mathcal{F}$-open. Of course $m \in G$ (because $m \in \bigcup \mu$ ), so $G \in \mathcal{E}_{m}$.

Now suppose that there is $H \in \mathcal{E}_{m}$ such that $H \notin \mathcal{F}_{m}$. It means that $w \notin H$ (a contradiction) or that $H \notin \mu$. If $H \cap[W \backslash \bigcup \mu] \neq \emptyset$, then we have a contradiction again: if there is any $n \in H \cap[W \backslash \bigcup \mu]$ and $H$ is $\mathcal{F}$-open, then it means that $n \in \mathcal{F} \operatorname{Int}(H)$, so $\mathcal{F}_{n} \neq \emptyset$. This is not possible because $n$ is even. 
Hence $H=\mathcal{F} \operatorname{Int}(H) \subseteq \bigcup \mu$. All the assumptions of Lemma 2.9 are satisfied. Thus $H \in \mu$. Of course, $m \in H$. It means that $H \in \mathcal{F}_{m}$. Finally, in this case $\mathcal{F}_{m}=\mathcal{E}_{m}$.

Note that the reasoning presented above is in fact general. Hence, we can formulate the following conclusion.

Theorem 5.3. Assume that $\langle W, \mu, \mathcal{F}\rangle$ is a $\mathbf{G T} \mathcal{F}$-structure and $\mathcal{F}_{w}=\emptyset$ for each $w \in W \backslash \bigcup \mu$. Then for any $v \in \bigcup \mu, \mathcal{F}_{v}=\mathcal{E}_{v}$. Moreover, this result is true also for any $w \in W \backslash \bigcup \mu: \mathcal{F}_{w}=\mathcal{E}_{w}=\emptyset$.

Now we can go further.

Definition 5.4. Let $\langle W, \mu, \mathcal{F}\rangle$ be a $\mathbf{G T} \mathcal{F}$ and let $\left(f_{\lambda}\right)$ be a gnet in $W$. We say that:

- $\left(f_{\lambda}\right) \mathcal{E}$-converges to $w \in W$ (i.e., $\left.\left(f_{\lambda}\right) \rightarrow^{\mathcal{E}} w\right)$ if for any $G \in \mathcal{E}_{w}, f_{\lambda}$ is eventually in $G$. In this case we say that $w$ is an $\mathcal{E}$-limit of $\left(f_{\lambda}\right)$. We say that $\left(f_{\lambda}\right)$ is $\mathcal{E}$-convergent if there is $v \in W$ such that $\left(f_{\lambda}\right) \rightarrow^{\mathcal{E}} v$;

- $w$ is an $\mathcal{E}$-limit point of $\left(f_{\lambda}\right)$ if it is frequently in every $G \in \mathcal{E}_{w}$.

What are the properties of such convergence? Let us start from constant gnets.

Lemma 5.5. Each constant gnet in any $\mathbf{G T} \mathcal{F}$-structure $\langle W, \mu, \mathcal{F}\rangle$ is $\mathcal{E}$ convergent.

Proof. Let us consider $\left(f_{\lambda}\right)=(w)$. Suppose that for any $v \in W,(w) \nrightarrow^{\mathcal{E}} v$. Hence, for any $v \in W$ there is $S \in \mathcal{E}_{v}$ such that $w \notin S$. In particular, this is true for $v=w$. Thus, there is $S \in \mathcal{E}_{w}$ such that $w \notin S$. This is impossible because of the very definition of $\mathcal{E}_{w}$.

Lemma 5.6. Let $\langle W, \mu, \mathcal{F}\rangle$ be a $\mathbf{G T} \mathcal{F}$ and $\left(f_{\lambda}\right)=(w)$ be a constant gnet in $W$. Then $(w)$ is $\mathcal{E}$-convergent if and only if $(w) \rightarrow^{\mathcal{E}} w$.

Proof. Assume that $(w) \nrightarrow^{\mathcal{E}} w$. It means that there is $S \in \mathcal{E}_{w}$ such that $w \notin S$. This is a contradiction.

Now we introduce the notion of $\mathcal{E} T_{1}$-spaces.

Definition 5.7. We say that a $\mathbf{G T} \mathcal{F}$-structure $\langle W, \mu, \mathcal{F}\rangle$ is $\mathcal{E} T_{1}$ if for any $w \neq v$ there are $G \in \mathcal{E}_{w}$ such that $v \notin G$ and $H \in \mathcal{E}_{v}$ such that $w \notin H$.

We can prove the following theorem about uniqueness.

Theorem 5.8. Let $\langle W, \mu, \mathcal{F}\rangle$ be a $\mathbf{G T} \mathcal{F}$-structure. Then the $\mathcal{E}$-limit of every constant gnet is unique if and only if $\langle W, \mu, \mathcal{F}\rangle$ is $\mathcal{E} T_{1}$.

Proof. Necessity. Suppose that $(w)$ is $\mathcal{E}$-convergent. We may assume that $(w) \rightarrow^{\mathcal{E}} w$. For any $v \neq w,(w) \nrightarrow^{\mathcal{E}} v$, i.e., there is $H \in \mathcal{E}_{v}$ such that $w \notin H$. 
But maybe for any $S \in \mathcal{E}_{w}, v \in S$ ? Let us consider the constant gnet $(v)$. Then $(v) \rightarrow^{\mathcal{E}} v$. But then $(v) \nrightarrow^{\mathcal{E}} w$. Hence there must be $G \in \mathcal{E}_{w}$ such that $v \notin G$.

Sufficiency. Assume that there is a constant gnet $(w)$ with two different $\mathcal{E}$-limits, i.e. $(w) \rightarrow^{\mathcal{E}} w$ and $(w) \rightarrow^{\mathcal{E}} v \neq w$. It means that for any $S \in \mathcal{E}_{v}$, $w \in S$, contradiction.

Below we prove a connection between convergence and $\mathcal{E}$-convergence.

Theorem 5.9. Let $\langle W, \mu, \mathcal{F}\rangle$ be a $\mathbf{G T} \mathcal{F}$ and $\left(f_{\lambda}\right)$ be a gnet (we assume that $f: P \rightarrow W)$. If $\left(f_{\lambda}\right) \rightarrow w$, then $\left(f_{\lambda}\right) \rightarrow^{\mathcal{E}} w$.

Proof. Suppose that $\left(f_{\lambda}\right) \nrightarrow^{\mathcal{E}} w$. Then there is $S \in \mathcal{E}_{w}$ such that for any $\lambda \in P$ there exists $\lambda_{1} \geq \lambda$ for which $f_{\lambda_{1}} \notin S$.

We know that $S \neq \emptyset$ (because $S \in \mathcal{E}_{w}$, so $w \in S$ ). Moreover, $S$ is $\mathcal{F}$-o., so $w \in \mathcal{F} \operatorname{Int}(S)$. Hence, there is $H \in \mathcal{F}_{w}$ such that $H \subseteq S$. Recall that $\left(f_{\lambda}\right) \rightarrow w$, so there is $\lambda_{0} \in P$ such that for any $\lambda \geq \lambda_{0}, f_{\lambda} \in H \subseteq S$. This is a contradiction.

We can easily prove that the converse of Theorem 5.9 is not true.

Example 5.10. Let us consider the $\mathbf{G T} \mathcal{F}$-structure $\langle W, \mu, \mathcal{F}\rangle$, where $W=\{w, v\}, \mu=\{\emptyset,\{w\}\}$ and $\mathcal{F}_{v}=\{\{w\}\}$. Then the set $\{w, v\}=W$ is $\mathcal{F}$-open and it is the only element of $\mathcal{E}_{v}$ (note that $\mathcal{F} \operatorname{Int}(\{v\})=\emptyset$ ). Now let us think about the constant gnet $\left(f_{\lambda}\right)=(v)$ (connected to an arbitrary $P)$. Undoubtedly, $(v) \rightarrow^{\mathcal{E}} v$. Note, however, that $(v) \nrightarrow v$ because there is $G \in \mathcal{F}_{v}$, namely $\{w\}$, such that $v \notin G$.

We can also reformulate Lemma 4.11. Now we do not need any special assumptions about $f_{m}$.

Lemma 5.11. Let $\langle W, \mu, \mathcal{F}\rangle$ be a $\mathbf{G T} \mathcal{F}$ and $f: P \rightarrow W$ be a gnet in $W$. Assume that $m$ is a maximal element of $P$. Then $\left(f_{\lambda}\right) \rightarrow^{\mathcal{E}} f_{m}$.

Proof. If $\mathcal{E}_{f_{m}}=\emptyset$, then the result is trivial. If not, then consider $S \in \mathcal{E}_{f_{m}}$. Clearly, $f_{m} \in S$ and $f_{\lambda} \in S$ for any $\lambda \geq m$ (because in such a case $\lambda=$ $m)$.

\section{Gnets and the question of closure}

6.1. Mutual dependence. There is a strict dependence between closures and gnets in generalized topology. It has been proven in [2] that if $\emptyset \neq A \subseteq$ $W$ and $w \in W$, then $w \in C l(A)$ if and only if there is a gnet $\left(f_{\lambda}\right)$ in $A$ (i.e., with its values in $A$ ) converging to $w$. However, the authors assumed that each point is in each of its neighbourhoods. Clearly, in our case this is false (for all points from $W \backslash \bigcup \mu$ ). For this reason, we formulate the following dependence. 
Theorem 6.1. Assume that $\langle W, \mu, \mathcal{F}\rangle$ is a $\mathbf{G T} \mathcal{F}$-structure, $\emptyset \neq A \subseteq W$. Then

$w \in \mathcal{F} C l(A) \Leftrightarrow$ there is a gnet $\left(f_{\lambda}\right) \in A$ such that $\left(f_{\lambda}\right) \rightarrow w$.

Proof. $(\Rightarrow)$ Assume that $w \in \mathcal{F} C l(A)$. There are two possibilites. First, $\mathcal{F}_{w}=\emptyset$. In this case we may assume that $P=2^{W} \backslash\{\emptyset\}$ and $C \geq D \Leftrightarrow C \subseteq D$. We define $f: P \rightarrow W$ in such a way that $f(C) \in A$. Clearly, $\left(f_{\lambda}\right)$ becomes a gnet in $A$ and moreover $\left(f_{\lambda}\right) \rightarrow w$ (because there are no sets in $\mathcal{F}_{w}$, so we can say anything about them).

Second option is that $\mathcal{F}_{w} \neq \emptyset$. Here we assume that $P=\mathcal{F}_{w}$. As for the $\geq$, it is defined as above. Note that (from the very definition of $\mathcal{F C l}(A)$ ) for any $G \in \mathcal{F}_{w}, G \cap A \neq \emptyset$. Then assume ${ }^{5}$ that $f(G) \in G \cap A$ for any $G \in \mathcal{F}_{w}$. Then $\left(f_{\lambda}\right)$ is a gnet in $A$ and for any $G \in \mathcal{F}_{w}$ our gnet is eventually in $G$, i.e., $\left(f_{\lambda}\right) \rightarrow w$.

$(\Leftarrow)$ Assume that there is a gnet $\left(f_{\lambda}\right)$ in $A$ such that $\left(f_{\lambda}\right) \rightarrow w$. Hence, for any $G \in \mathcal{F}_{w},\left(f_{\lambda}\right)$ is eventually in $G$, which means that for any $G \in \mathcal{F}_{w}$ there is $\lambda_{0}$ such that for any $\lambda \geq \lambda_{0}, f_{\lambda} \in G$. But for any $\lambda, f_{\lambda} \in A$. Hence, for any $G \in \mathcal{F}_{w}, G \cap A \neq \emptyset$. Thus $w \in \mathcal{F} C l(A)$. Moreover, due to Lemma 2.26, $w \in C l(A)$.

Is it possible to replace $\rightarrow$-convergence by $\rightarrow^{\mathcal{E}}$-convergence? Of course, if $w \in \mathcal{F} C l(A)$, then we can find our expected $\rightarrow$-convergent gnet, as it has been shown above: and this gnet is (by means of Lemma 5.6) $\rightarrow^{\mathcal{E}_{\text {- }}}$ convergent. But the converse is not true. Let us think about the following (counter)-example.

Example 6.2. Let $\langle W, \mu, \mathcal{F}\rangle$ be a $\mathbf{G T} \mathcal{F}$-structure where $W=\{w, v, u\}$, $\mu=\{\emptyset,\{w\}\}, \mathcal{F}_{v}=\{\{w\},\{u\}\}, \mathcal{F}_{u}=\emptyset$. Of course, $\mathcal{F}_{w}=\{\{w\}\}$. Consider $A=\{v\}$ and the constant gnet $(v)$. Clearly, $(v)$ is a gnet in $A$. It is $\rightarrow^{\mathcal{E}_{-}}$ convergent (at least to $v$ ).

Now $v \notin \mathcal{F} C l(A)$ because there is $G=\{u\} \in \mathcal{F}_{v}$ such that $G \cap\{v\}=$ $G \cap A=\{u\} \cap\{v\}=\emptyset$. In fact, $\mathcal{F} C l(A)=\mathcal{F} C l(\{v\})=\{z \in W$; for any $G \in$ $\left.\mathcal{F}_{z}, G \cap\{v\} \neq \emptyset\right\}=\{u\}$ (because there are no sets in $\mathcal{F}_{u}$ ).

6.2. $\mathcal{E}$-open sets and their generalized topology. We have introduced $\mathcal{F}$-interiors (closures) to speak later about $\mathcal{F}$-open (closed) sets. Then we have discussed the set $\mathcal{E}_{w}$ for an arbitrary $w$. One could ask: does it make sense to move these notions on even more high level? We wish to treat this issue very briefly.

Definition 6.3. Assume that $\langle W, \mu, \mathcal{F}\rangle$ is a $\mathbf{G T} \mathcal{F}$-structure and $A \subseteq W$. We say that:

\footnotetext{
${ }^{5}$ This reasoning is based on the one for ordinary generalized neighbourhoods, presented in [2]. However, there is a mistake there (probably a typo). The authors assumed only that $f(G) \in G$ (we use our notation). Clearly, we must assume that our gnet is in the (nonempty) intersection of a neighbourhood and the set $A$.
} 
- $w \in \mathcal{E} \operatorname{Int}(A)$ if there is $S \in \mathcal{E}_{w}$ such that $S \subseteq A$;

- $w \in \mathcal{E} C l(A)$ if for any $S \in \mathcal{E}_{w}, S \cap A \neq \emptyset$.

We say that $A$ is $\mathcal{E}$-open if $\mathcal{E} \operatorname{Int}(A)=A$ and that $A$ is $\mathcal{E}$-closed if $\mathcal{E} C l(A)=$ $A$.

Although at this stage of research such definitions seem to be somewhat artificial, there is at least one interesting thing to note.

Theorem 6.4. Let $\langle W, \mu, \mathcal{F}\rangle$ be a $\mathbf{G T} \mathcal{F}$-structure and let $\mathcal{E O}$ be a collection of all $\mathcal{E}$-open sets (with respect to $\mu$ and $\mathcal{F}$ ). Then $\mathcal{E} \mathcal{O}$ forms a generalized topology on $W$. If for any $w \in W, \mathcal{F}_{w} \neq \emptyset$, then $\mathcal{E} \mathcal{O}$ is a strong generalized topology.

Proof. First, let us prove that $\emptyset$ is $\mathcal{E}$-open. Compute: $\mathcal{E} \operatorname{Int}(\emptyset)=\{z \in$ $W$; there is $S \in \mathcal{E}_{w}$ such that $\left.S \subseteq \emptyset\right\}=\emptyset$. It is because the only set contained in $\emptyset$ is the empty set itself, but for any $S \in \mathcal{E}_{w}, w \in S$ and hence $S \neq \emptyset$.

Assume now that $J \neq \emptyset$ and for any $i \in J, X_{i}$ is $\mathcal{E}$-open. Then $\bigcup X_{i}$ is also $\mathcal{E}$-open, i.e., $\mathcal{E} \operatorname{Int}\left(\bigcup_{i \in J} X_{i}\right)=\bigcup X_{i}$.

$(\subseteq)$ Let $w \in \mathcal{E} \operatorname{Int}\left(\bigcup X_{i}\right)$. Hence there is $S \in \mathcal{E}_{w}$ such that $S \subseteq \bigcup_{i \in J} X_{i}$. But $w \in S$. Thus $w \in \bigcup_{i \in J} X_{i}$.

() Let $w \in \bigcup_{i \in J} X_{i}$. Then there is $X_{k}$ such that $w \in X_{k} . X_{k}$ is $\mathcal{E}$-open, so $w \in \mathcal{E} \operatorname{Int}\left(X_{k}\right)$. Thus there is $S \in \mathcal{E}_{w}$ such that $S \subseteq X_{k} \subseteq \bigcup_{i \in J} X_{i}$. Hence $w \in \mathcal{E} \operatorname{Int}\left(\bigcup_{i \in J} X_{i}\right)$.

Now assume that $\mathcal{F}_{w} \neq \emptyset$ for any $w \in W$. Then $\mathcal{E}_{w} \neq \emptyset$. Hence $\mathcal{E} \operatorname{Int}(W)=\left\{z \in W\right.$; there is $S \in \mathcal{E}_{w}$ such that $\left.S \subseteq W\right\}=W$.

Informally speaking, even if we are working with $\mathbf{G T} \mathcal{F}$ which is not strong (but for any $w \in W, \mathcal{F}_{w} \neq \emptyset$ ), we still can find a generalized topology (namely, $\mathcal{E} \mathcal{O}$ ) which depends on $\mathcal{F}$ and "covers" each element of $W$.

\section{Acknowledgements}

We are grateful to the anonymous reviewer for valuable comments which helped us to improve the quality of this paper.

\section{References}

[1] H. Ahmet and T. Mehmet, Peritopological spaces and bisimulations, Rep. Math. Logic 50 (2015), 67-81.

[2] R. Baskaran, M. Murugalingam, and D. Sivaraj, Generalized nets in generalized topological spaces, J. Adv. Res. Pure Math. 3(2) (2011), 49-55.

[3] R. Baskaran, M. Murugalingam, and D. Sivaraj, Sequential convergence in generalized topological spaces, J. Adv. Res. Pure Math. 3(1) (2011), 111-122.

[4] G. Choquet, Convergences, Ann. Univ. Grenoble, Sect. Sci. Math. Phys. (N. S.) 23 (1948), 57-112.

[5] Á. Császár, Generalized open sets, Acta Math. Hungar. 75 (1997), 65-87. 
[6] Á. Császár, Generalized topology, generalized continuity, Acta Math. Hungar. 96(4) (2002), 351-357.

[7] Á. Császár, Separation axioms for generalized topologies, Acta Math. Hungar. 104(12) (2004), 63-69.

[8] Á. Császár, Generalized open sets in generalized topologies, Acta Math. Hungar. 106 (2005), 53-66.

[9] A. P. Dhana Balan and P. Padma, Separation spaces in generalized topology, Int. J. Math. Res. 9(1) (2017), 65-74.

[10] J. P. Doignon and J. C. Falmagne, Knowledge spaces and learning spaces, in: New Handbook of Mathematical Psychology. Vol. 1, Cambridge University Press, Cambridge, 2017, pp. 274-321.

[11] M. Hussain, M. ud Din Khan, and C. Özel, On generalized topological groups, Filomat $\mathbf{2 7}(4)$ (2013), 567-575.

[12] J. Järvinen, M. Kondo, and J. Kortelainen, Logics from Galois connections, Internat. J. Approx. Reason. 49(3) (2008), 595-606.

[13] E. Korczak-Kubiak, A. Loranty, and R. J. Pawlak, Generalized (topological) metric spaces. From nowhere density to infinite games, http://dspace.uni.lodz.pl: 8080/xmlui/bitstream/handle/11089/24747/89-104-korczak.pdf?sequence=1\& isAllowed $=y$.

[14] A. S. Masshour, A. A. Allam, F. S. Mahmoud, and F. H. Khedr, On supratopological spaces, Indian J. Pure Appl. Math. 14(4) (1983), 502-510.

[15] G. E. Moore, The New Haven Mathematical Colloquium, Editors E. H. Moore, E. J. Wilczynski, and Max Mason, Yale University Press, 1910.

[16] S. Palaniammal and M. Murugalingam, Generalized filters, Int. Math. Forum 9(36) (2014), 1751-1756.

[17] A. Piekosz, Generalizations of topological spaces, https://www.researchgate.net/ publication/314392123_Generalizations_of_Topological_Spaces.

[18] R. D. Sarma, On convergence in generalized topology, Int. J. Pure Appl. Math. 60(2) (2010), 205-210.

[19] M. S. Sarsak, New separation axioms in generalized topological spaces, Acta Math. Hungar. 132(3) (2011), 244-252.

[20] H. Soldano, A modal view on abstract learning and reasoning, in: Ninth Symposium on Abstraction, Reformulation, and Approximation, SARA, 2011, pp. 99-106.

[21] J. C. C. McKinsey and A. Tarski, The algebra of topology, Ann. of Math. 45(1) (1944) $141-191$

[22] T. Witczak, Generalized topological semantics for weak modal logics, https://arxiv. org/pdf/1904.06099.pdf.

Institute of Mathematics, Faculty of Science and Technology, University of Silesia, Bankowa 14, 40-007 Katowice, Poland

E-mail address: tm.witczak@gmail.com 